\title{
Educação médica em terapêuticas não convencionais
}

\author{
Medical education in non-conventional therapies
}

Marcus Zulian Teixeira ${ }^{1}$, Chin An Lin ${ }^{2}$

Teixeira MZ, Lin CA. Educação médica em terapêuticas não convencionais / Medical education in non-conventional therapies. Rev Med (São Paulo). 2013 out.-dez.;92(4):224-35.

RESUMO: A demanda da população pela medicina alternativa e complementar (CAM) vem aumentando substancialmente nas últimas décadas, exigindo dos médicos noções básicas dessas diversas terapias, a fim de que possam orientar os pacientes em tratamentos diferentes dos que estão habituados a prescrever. Assim sendo, compete às escolas de medicina propiciar aos estudantes o conhecimento das evidências científicas, dos pressupostos teóricos e das abordagens práticas empregadas por estas distintas formas de tratamento. A presente revisão tem o propósito de fomentar a discussão sobre o ensino de terapêuticas não convencionais nas escolas de medicina, enfocando o interesse da população e da classe médica, a importância das iniciativas na graduação e na pós-graduação (residência), e as perspectivas da educação médica em terapêuticas não convencionais.

DESCRITORES: Educação médica; Terapias complementares; Homeopatia; Acupuntura; Currículo.

\begin{abstract}
The demand of the population by complementary and alternative medicine (CAM) has been substantially increasing in the last decades, requiring that doctors have basic notions of these various therapies, in order to guide patients in different treatments of those who are accustomed to prescribe. In this way, the medical schools have the responsibility to provide to students the knowledge of scientific evidences, theoretical assumptions and practical approaches used in these different forms of treatment. The present review has the purpose of fomenting the discussion regarding the teaching of non-conventional therapeutics in medical schools. It is focused on the interest of the population and the medical class, the importance of the initiatives in undergraduate and graduate (residency), and the perspectives of medical education in non-conventional therapeutics.
\end{abstract}

KEY WORDS: Education, medical; Complementary therapies; Homeopathy; Acupuncture; Curriculum.

1. MD, PhD. Coordenador da disciplina optativa "Fundamentos da Homeopatia" (MCM0773) da Faculdade de Medicina da Universidade de São Paulo (FMUSP)

2. MD, PhD. Professor Colaborador do Departamento de Clínica Médica da FMUSP. Docente da disciplina optativa "Introdução à Acupuntura" (MSP0668) da FMUSP

Endereço para correspondência: Marcus Zulian Teixeira. Hospital das Clínicas da FMUSP. Serviço de Clínica Médica Geral. Av. Dr. Enéas de Carvalho Aguiar, 155, $4^{\circ}$ andar - bloco 6. CEP: 05403-900. São Paulo, SP. E-mail: mzulian@usp.br 


\section{INTRODUÇÃO}

D e forma alternada, complementar ou integrada ao modelo biomédico convencional, o emprego de terapêuticas não convencionais vêm aumentando substancialmente nas últimas décadas, sendo utilizadas em todos os países e por todas as camadas da população. Esse panorama exige do médico noções básicas dessas terapias, a fim de que possa orientar os pacientes que desejem utilizar tratamentos distintos dos que está habituado a prescrever.

Em vista dos milhões de americanos $(40 \%$ da população) que utilizam alguma forma de terapêutica não convencional, o National Institutes of Health $(\mathrm{NIH}$, EUA) possui uma agência exclusivamente dedicada à investigação, divulgação e controle destas práticas (National Center for Complementary and Alternative Medicine NCCAM $)^{1}$, definindo este conjunto de terapias pelo termo "complementary health approaches" ou "complementary and alternative medicine" (CAM). Pela sigla CAM ser o termo mais citado nos estudos e pesquisas, iremos utilizála nesta revisão.

No Brasil, apesar do Conselho Federal de Medicina (CFM) reconhecer a homeopatia e a acupuntura como especialidades médicas desde 1980 e 1995, respectivamente, elas estão disponibilizadas na rede pública e privada de saúde de forma irrelevante e insuficiente à demanda da população. Paradoxalmente, levantamento recente realizado pelo Conselho Regional de Medicina do Estado de São Paulo (CREMESP) e pelo CFM, mosttrou que a acupuntura e a homeopatia ocupam, respectivamente, o $22^{\circ} \mathrm{e}$ o $28^{\circ}$ contingente de profissionais dentre 53 especialidades médicas brasileiras ${ }^{2}$.

Em consequência da ausência do ensino regular destas abordagens no currículo das escolas de medicina, a classe médica não está apta para discorrer sobre as diversas formas de CAM junto à população, criando um hiato na terapêutica e/ou na relação médico-paciente. Por si só, estas evidências deveriam estimular as escolas médicas a propiciarem aos graduandos, pós-graduandos e residentes o conhecimento dos fundamentos teóricos, das evidências científicas e das técnicas empregadas por estas formas de tratamento. Além disso, a incorporação efetiva de práticas reconhecidas junto aos serviços de saúde, atuando de forma adjuvante e complementar às terapêuticas convencionais, ampliaria a eficiência, a eficácia e a efetividade do ato médico nas diversas especialidades e áreas de atuação.

Com o intuito de dar continuidade ao debate sobre a necessidade de incorporação do ensino de CAM nas escolas de medicina ${ }^{3-7}$, elaboramos esta revisão atualizada sobre a validade do ensino na graduação e na pós-graduação (residência), as atitudes da população e da classe médica, as iniciativas do ensino acadêmico em diversos países e os benefícios que o aprendizado destas terapêuticas pode trazer à população e à formação médica.

\section{VALIDADE DO ENSINO DE CAM NA GRADUAÇÃO MÉDICA}

\section{Interesse e emprego de CAM pela população mundial}

No primeiro levantamento (1990) sobre a prevalência, os custos e as formas de utilização de CAM nos EUA, Eisenberg et al. ${ }^{8}$ estimaram que $34 \%$ da população adulta americana a empregava, perfazendo um total de 427 milhões de consultas/ano com terapeutas não médicos. Em 1997 repetiram a pesquisa ${ }^{9}$, notando um incremento na procura por CAM ( $42 \%$ da população, com 629 milhões de consultas/ano), que representava um custo adicional de US\$ 27 bilhões à população americana, por não estarem disponíveis nos serviços públicos de saúde e não serem reembolsadas pelas empresas de seguro médico. Novo levantamento em $2002^{10}$ mostrou que a prevalência do uso de CAM permanecia constante, estimando o emprego de duas ou mais modalidades por 72 milhões de americanos adultos. Pesquisa realizada na Europa ${ }^{11}$ mostrou resultados semelhantes, com $46 \%$ da população alemã e $49 \%$ da população francesa fazendo uso de CAM.

Levantamento com moradores da Flórida (EUA, $1998)^{12}$ evidenciou que $62 \%$ afirmaram ter utilizado uma ou mais de 11 modalidades citadas, com maior prevalência dos remédios caseiros (31\%), dietas especiais (24\%), técnicas de relaxamento $(20 \%)$ e fitoterapia $(18 \%)$. Em $2002^{10}$, a terapêutica mais empregada era a fitoterapia $(18,6 \% ; 38$ milhões de americanos), seguida pelas técnicas de relaxamento $(14,2 \%$; 29 milhões de americanos) e quiropraxia $(7,4 \% ; 15$ milhões de americanos). Em conformidade com levantamento abrangente e recente sobre a utilização de CAM pelos americanos (National Health Interview Survey, 2007) ${ }^{1,13}$, as dez modalidades mais utilizadas pela população adulta são: produtos naturais $(17,7 \%)$, respiração profunda $(12,7 \%)$, meditação $(9,4 \%)$, quiropraxia e osteopatia $(8,6 \%)$, massagens $(8,3 \%)$, yoga $(6,1 \%)$, dietas $(3,6 \%)$, relaxamento profundo $(2,9 \%)$, imaginação guiada $(2,2 \%)$ e homeopatia $(1,8 \%)$.

Pesquisas sobre os motivos que levavam a população americana a procurar terapêuticas não convencionais mostrou que a insatisfação dos pacientes com a medicina ortodoxa era a principal justificativa ${ }^{14,15}$. Outra justificativa estava na busca por uma compreensão holística para o fenômeno do adoecer (intercorrelação corpo-mente-espírito) ${ }^{16}$. De forma análoga, estudos brasileiros ${ }^{17,18}$ mostraram que os pacientes procuravam a homeopatia devido aos seguintes motivos: insatisfação com a medicina convencional, evitar os efeitos colaterais das drogas clássicas, incremento na relação médico-paciente e tratamento que englobe a pessoa como um todo (corpo-mente-espírito).

\section{Importância do aprendizado em CAM para a classe médica}

No segundo levantamento ${ }^{9}$ com a população 
americana, constatou-se que acima de $60 \%$ dos pacientes que utilizavam CAM não relatava este fato aos seus médicos. Assim como em outras doenças, pesquisas realizadas junto a pacientes com câncer de mama mostraram que elas evitavam discutir o uso concomitante da CAM com seus médicos, antecipando uma resposta reprovadora, em vista do descrédito e da falta de conhecimento no assunto dos profissionais ${ }^{19,20}$. A ausência do interesse médico pelo possível uso complementar de CAM pode significar um risco para os pacientes, em consequência de interações medicamentosas ou efeitos adversos ${ }^{21,22}$.

A maioria da classe médica também não está apta a responder perguntas ou orientar seus pacientes quanto ao uso de CAM, no que tange aos mecanismos de ação, indicações terapêuticas, interações medicamentosas e efeitos adversos destas terapêuticas ${ }^{22,23}$. Fatores adicionais como o diálogo insuficiente entre médicos convencionais e não convencionais, dúvidas da competência dos profissionais e o risco de oferecimento de esperança irreal de cura, colocam o paciente numa posição incerta frente às terapêuticas não convencionais. A incorporação sistemática de informações sobre CAM nos currículos médicos, além de diminuir o preconceito existente, proveria aos futuros médicos o conhecimento necessário para permitir que seus pacientes possam se beneficiar adequadamente da $\mathrm{CAM}^{5,24-30}$.

Por outro lado, a exploração de tópicos de CAM no currículo médico promoveria um foco humanizante e salutogênico nos cuidados à saúde ${ }^{6,31}$, elucidando a natureza ampla, complexa e incerta da prática médica, desenvolvendo outras habilidades nas tomadas de decisões clínicas e provendo novos conhecimentos para futuras pesquisas $^{32-34}$.

O emprego de CAM nas doenças crônicas e enfermidades pouco responsivas aos tratamentos convencionai ${ }^{35-38}$ poderia incrementar o arsenal terapêutico da medicina moderna. Em diversas iniciativas ${ }^{39-41}$, a integração entre práticas convencionais e não convencionais evidenciou aumento na qualidade do atendimento e melhora na relação custo/efetividade.

\section{ATITUDES DA CLASSE MÉDICA PERANTE CAM}

\section{Atitudes dos médicos}

O desconhecimento dos fundamentos básicos destas abordagens pela classe médica causa frustração nos pacientes que optaram pela sua utilização em concomitância ao tratamento convencional, privando-os de uma orientação segura sobre as principais indicações e os possíveis riscos dessas abordagens ${ }^{42-44}$.

Com o aumento do interesse da população por CAM, os médicos sentem a necessidade de suprir esta demanda, desviada nos EUA para os terapeutas não médicos. Unida ao desgosto dos pacientes pelo sistema de saúde convencional, a insatisfação dos médicos pelo mesmo modelo tem ocasionado aumento no interesse por estas práticas complementares ${ }^{45,46}$.

Metanálise de 12 levantamentos das atitudes de médicos convencionais frente às abordagens complementares em saúde mostrou que eles as consideravam moderadamente efetivas ${ }^{47}$. Pesquisa sobre as atitudes de médicos generalistas de Victoria (Austrália) ${ }^{48}$ perante o uso de CAM mostrou que acupuntura, hipnose e meditação são bem aceitas pelos mesmos, tendo sido citadas por $80 \%$ dos seus pacientes e utilizadas por $50 \%$. Os médicos referiram treinamento em várias práticas: meditação (34\%), acupuntura (23\%), terapia vitamínica-mineral (23\%), hipnose $(20 \%)$, medicina herbária $(12 \%)$, quiroprática $(8 \%)$, naturopatia $(6 \%)$, homeopatia $(5 \%)$, cura espiritual $(5 \%)$, osteopatia (4\%), aromaterapia (4\%) e reflexologia $(2 \%)$. Em torno de $30 \%$ dos entrevistados manifestaram interesse em aprender quiroprática, medicina herbária, naturopatia e terapia vitamínica-mineral.

Levantamento junto a médicos de Denver (Colorado, USA $)^{49}$ sobre a experiência pessoal e com pacientes frente à CAM evidenciou que $76 \%$ informaram ter pacientes que as usavam, 59\% eram questionados sobre modalidades específicas, $48 \%$ tinham recomendado a pacientes e $24 \%$ tinham empregado em si próprios. Poucos médicos sentiam-se confortáveis em discutir CAM com seus pacientes e a maioria (84\%) acreditava que precisaria aprender mais para responder às dúvidas dos pacientes adequadamente.

Pesquisa sobre as atitudes, o conhecimento e o interesse de pediatras americanos (Michigan, USA) perante a $\mathrm{CAM}^{50}$ revelou que acima de $50 \%$ estavam interessados em frequentar cursos de formação, utilizariam pessoalmente e indicariam a seus pacientes, dando preferência ao biofeedback $(23,6 \%)$, grupos de auto-ajuda $(23,3 \%)$, relaxamento $(14,9 \%)$, hipnose $(13,8 \%)$ e acupuntura ou acupressura (10,9\%). Outros levantamentos, em diversas localidades e com médicos de distintas especialidades (cirurgiões, oncologistas, etc.) mostraram interesses semelhantes no uso e capacitação em $\mathrm{CAM}^{51-56}$.

\section{Atitudes de estudantes de medicina e médicos residentes}

Levantamento com estudantes de medicina da Universidade de Düsseldorf (Alemanha) ${ }^{57}$ revelou que os entrevistados apresentavam conhecimento das práticas, experiência como pacientes e interesse em aprender uma ou mais técnicas de CAM. O maior interesse no aprendizado foi com acupuntura $(55,7 \%)$, homeopatia $(42,1 \%)$, treinamento autógeno $(24,9 \%)$ e reflexologia $(11,4 \%)$, acreditando serem também as mais eficazes. Pesquisa realizada com 800 estudantes de medicina de duas universidades de Melbourne (Austrália) ${ }^{58}$ revelou atitudes positivas perante CAM com desejo na aprendizagem, embora apresentassem pouco conhecimento sobre o tema. Levantamento com estudantes 
de medicina de Singapura ${ }^{59}$ mostrou atitudes positivas em relação à CAM: $92 \%$ acreditavam que estas abordagens incluem idéias e métodos que podem beneficiar a medicina convencional, $86 \%$ desejavam adquirir conhecimento e 91\% afirmavam que a CAM teria um papel importante em sua futura prática médica.

De forma análoga, pesquisa com alunos do $1^{\circ} \mathrm{e} 2^{\circ}$ anos da escola de medicina da Universidade Georgetown (Washington, EUA) ${ }^{60}$ evidenciou que a maioria dos estudantes (91\%) concordava que "CAM inclui idéias e métodos que poderia beneficiar a medicina", acima de $85 \%$ concordavam que "o conhecimento sobre CAM é importante para um futuro profissional da saúde", e acima de $75 \%$ referiam que "CAM deveria ser incluída no currículo". O nível de formação mais desejado foi para "aconselhar pacientes sobre o uso". O maior nível era procurado para acupuntura, quiropraxia, fitoterapia e suplementos nutricionais. Outros levantamentos mostraram atitudes semelhantes ${ }^{61-64}$.

Levantamento das atitudes dos estudantes de medicina da Faculdade de Medicina da Universidade de São Paulo (FMUSP) ${ }^{4}$ perante a homeopatia e a acupuntura, na média das duas disciplinas, mostrou que acima de $85 \%$ dos estudantes considerava que elas deveriam estar inseridas no currículo da graduação das escolas médicas, com 56\% dos entrevistados mostrando-se bastante interessados no aprendizado. Apesar de nenhum ou pouco conhecimento no assunto $(76 \%), 67 \%$ acreditavam em algum grau de eficácia, tendo como principais indicações as doenças crônicas, isoladamente (37\%) ou englobando também as doenças agudas (29\%). Ao redor de 35\% dos acadêmicos foram favoráveis ao oferecimento ambulatorial de ambas as especialidades nos serviços públicos de saúde, enquanto a média de $34 \%$ defendia a oferta destes tratamentos também em hospitais, com $60 \%$ acreditando na possibilidade de integração com a medicina convencional.

Buscando avaliar o programa de quatro anos da residência em medicina familiar integrativa da Universidade de Washington (EUA), que associa CAM ao currículo convencional, pesquisa realizada junto a 39 médicos residentes do $3^{\circ}$ e $4^{\circ}$ anos mostrou que $80 \%$ dos entrevistados consideravam que a residência deveria prover treinamento em CAM, sendo que a maioria já havia recomendado alguma prática de CAM aos pacientes no último ano ${ }^{65}$. Levantamento junto a 153 médicos residentes em medicina de família no Arkansas (EUA) ${ }^{66}$ evidenciou conhecimento mínimo no assunto, ausência de questionamento aos pacientes sobre o uso dessas abordagens e desconforto ao aconselhar seus pacientes sobre os riscos e benefícios; no entanto, a maioria estava interessada em aprender CAM.

A Association of American Medical Colleges (EUA) declarou que estudantes de medicina deveriam ter conhecimento suficiente sobre CAM para que, futuramente, estejam aptos a aconselhar seus pacientes a respeito dos possíveis benefícios e riscos de cada terapêutica ${ }^{67}$.

\section{PANORAMA DA EDUCAÇÃO MÉDICA EM CAM}

Em resposta ao interesse crescente pelo aprendizado da CAM, escolas médicas e programas de pós-graduação e residência médica começam a incluir o ensino dessas abordagens em seus currículos, após terem percebido que com esta atitude aumentam a amplitude de ação da medicina e melhoram a relação médico-paciente ${ }^{68}$.

No Reino Unido, a homeopatia dispõe de um estatuto legal que permite a formação pós-graduada de médicos. Em 1993, a British Medical Association ${ }^{69}$ passou a recomendar que as escolas médicas oferecessem cursos de introdução em CAM para todos acadêmicos. Três anos após $(1996)^{70}, 23 \%$ das faculdades de medicina do Reino Unido haviam incorporado ao currículo disciplinas que ministravam conceitos básicos sobre CAM e, em 1999, $40 \%$ das escolas médicas da União Européia ofereciam cursos de $\mathrm{CAM}^{71}$. Em 1997, a Ordem dos Médicos da França reconheceu que a homeopatia deveria ser prescrita por médicos que recebecem formação ao nível da pósgraduação universitária.

Em alguns estados americanos (Arizona, Nevada e Connecticut) existem autoridades de certificação para homeopatas. O American Institute of Homeopathy confere diploma de homeoterapeuta para médicos e o Council of Homeopathic Certification oferece certificação para a homeopatia clássica. A licença para médicos acupunturistas é oferecida em alguns estados americanos. Acompanhando as mudanças exigidas pela população americana, a última versão do Código de Ética Médica do American College of Physicians incluiu uma seção específica sobre "tratamentos alternativos", recomendando que o médico deva acompanhar o paciente se este decide fazer um tratamento não convencional ${ }^{72}$.

Nos EUA, é grande o número de escolas médicas que oferecem aulas isoladas em medicina holística ou $\mathrm{CAM}^{73}$. Levantamento realizado em 1995 pela Society of Teachers of Family Medicine junto a 97 escolas médicas dos EUA mostrou que 39,2\% ofereciam alguma forma de instrução em CAM aos seus residentes, em sua maioria na forma de disciplinas optativas $(72,2 \%)$. Nas residências não universitárias para médicos de família, $28,1 \%$ ofereciam instrução em $\mathrm{CAM}^{74}$.

No período de 1997-98, uma pesquisa em 117 escolas médicas americanas mostrou que $64 \%$ ministravam aulas sobre $\mathrm{CAM}^{75}$. Em 1998, levantamento realizado junto a escolas médicas do Canadá mostrou que $81 \%$ delas haviam incorporado tópicos de CAM ao seu currículo, estando a acupuntura e a homeopatia como as mais frequentes ${ }^{76}$. No período 1998-99, uma pesquisa junto a 80 escolas médicas japonesas mostrou que $20 \%$ ensinavam CAM, prevalecendo a acupuntura ${ }^{77}$.

Outro levantamento sobre a situção do ensino 
de CAM nos EUA $^{78}$ foi respondido por 73 diretores de cursos (53 escolas médicas americanas). Os tópicos mais frequentemente ensinados eram acupuntura $(76,7 \%)$, fitoterapia $(69,9 \%)$, meditação e relaxamento $(65,8 \%)$, espiritualismo/religião/oração $(64,4 \%)$, quiroprática $(60,3 \%)$, homeopatia $(57,5 \%)$ e nutrição/dietas $(50,7 \%)$. Apesar de alguns receberem mais tempo de instrução, a carga horária média era de duas horas-aula por tópico. Os cursos 'típicos' em CAM estavam sob a responsabilidade dos Departamentos de Clínica Médica (64,9\%), sendo oferecidos na forma de disciplina eletiva $(75,3 \%)$, do primeiro ao quarto ano da graduação, contemplando menos do que 20 horas-aula de instrução $(52,1 \%)$. O ensino da maioria dos cursos $(78,1 \%)$ era feito por diversos indivíduos identificados como práticos ou prescritores de CAM. A maioria dos cursos tinha como objetivo principal ensinar conceitos gerais de CAM $(61,6 \%)$ e uma minoria enfatizava as evidências científicas e a efetividade das mesmas $(17,8 \%)$, ou oferecia treinamento prático no uso de técnicas específicas (17,8\%).

No Brasil, apesar de a homeopatia e a acupuntura serem reconhecidas como especialidades médicas, elas estão incorporadas ao currículo acadêmico em poucas faculdades de medicina, sendo oferecidas, principalmente, na forma de disciplinas eletivas (optativas).

\section{PROPOSTAS DA EDUCAÇ̃̃O MÉDICA EM CAM}

\section{Graduação médica}

$\mathrm{Na}$ Alemanha foi criado um projeto que buscava integrar os 'processos naturais de cura' na pesquisa e no ensino da Universidade Ludwig-Maximilian de Munique $^{79}$. O curso opcional para os estudantes de medicina incluia aulas sobre os fundamentos básicos e as pesquisas desenvolvidas em acupuntura, terapia manual, nutrição, homeopatia, hidroterapia e fitoterapia, abordando as evidências científicas sobre os efeitos e a eficácia, indicações e contra-indicações de cada terapêutica, além de treinamento prático em certas práticas.

Em Taiwan ${ }^{80}$ e no Japão ${ }^{81}$ surgiram propostas para integrar a medicina ocidental à medicina oriental (medicina chinesa) a partir do ensino médico fundamental, acreditando-se que um sistema de cuidados médicos unificado diminuiria os custos gerais com a saúde.

A Universidade do Arizona ${ }^{82}$, pioneira na educação médica em CAM nos EUA desde 1983, oferece aos residentes e estudantes do quarto ano da graduação, desde 1997, uma disciplina eletiva em "medicina integrativa" com quatro semanas de duração, aprofundando os temas abordados superficialmente nos primeiros anos do curso e propiciando uma vivência clínica. Propostas semelhantes ocorreram em outras escolas médicas ${ }^{83,84}$.

Segundo o National Center for Complementary and Alternative Medicine (NCCAM-NIH), o ensino de CAM para acadêmicos de medicina deveria estar pautado nos fundamentos básicos de cada modalidade, enfatizando a necessidade do embasamento científico para as diversas condutas terapêuticas ${ }^{85}$. Diferentes níveis de competência em CAM são reconhecidos nos EUA: a) baixo, em que os médicos possuem conhecimentos básicos para indicar e encaminhar seus pacientes a outros profissionais mais qualificados; b) médio, em que a habilidade prática permite que os médicos atuem em condições e patologias específicas; c) alto, em que a competência lhes dá a habilidade para tratar diversas doenças.

$\mathrm{Na}$ década passada, o NCCAM-NIH financiou o Projeto de Educação em Medicina Alternativa e Complementar, com propostas que visavam integrar a CAM aos currículos das escolas de medicina, fomentando a formação de profissionais para atender à demanda da população. Com esta iniciativa, diversos benefícios foram gerados: aumento das atividades acadêmicas na área, criação de novos programas e aumento das colaborações intra e inter-universidades. Desafios comuns incluíram a necessidade de professores qualificados, mudanças e adensamentos dos currículos, falta de definição nas práticas de CAM e sustentabilidade futura aos programas ${ }^{86,87}$.

Iniciativa semelhante surgiu no Canadá, com o objetivo de criar programas para integrar CAM na graduação médica. As atividades do projeto incluíam a elaboração de competências específicas na área, revisões de tópicos relevantes, repositório de recursos de ensino e aprendizagem, e um guia para o desenvolvimento, implantação e sustentabilidade do currículo em $\mathrm{CAM}^{88}$.

Um consórcio constituído por dezenas de escolas médicas dos EUA, Canadá e México com programas ativos no ensino de CAM e medicina integrativa tem reunido esforços para incorporar estas abordagens ao currículo das escolas e residências médicas. O Consortium of Academic Health Centers for Integrative Medicine (CAHCIM) ${ }^{89}$ tem influenciado o National Board of Medical Examiners (EUA) para incluir nas suas provas de avaliação questões sobre CAM e medicina integrativa.

Levantamento recente realizado junto a 41 escolas médicas da Coréia $(2007-2010)^{90}$ revelou que CAM era ensinada oficialmente em 35 escolas $(85,4 \%)$ na forma de cursos de introdução à CAM ou medicina integrativa $(88,6 \%)$, medicina coreana tradicional $(57,1 \%)$, homeopatia/naturopatia $(31,4 \%)$ e acupuntura $(28,6 \%)$. $\mathrm{O}$ formato educacional incluía palestras com professores e demonstrações com praticantes. A ordem de valor das principais competências foi atitude (40/41), conhecimento (32/41) e habilidade (6/41).

No Brasil, a homeopatia e a acupuntura são ensinadas na forma de disciplinas eletivas (60 horas-aula, 4 créditos $)^{91,92} \mathrm{em}$ poucas faculdades de medicina, estando sua incorporação ao currículo na dependência da vontade política de coordenadores de cursos e/ou diretores dessas instituições, sendo ministradas, comumente, de forma 
voluntária por médicos especialistas nas áreas ${ }^{3,5,7,93}$.

\section{Residência médica e pós-graduação}

Nos EUA, a Society of Teachers of Family Medicine Group on Alternative Medicine desenvolveu um consenso de recomendações sobre atitudes, conhecimentos e habilidades em CAM para serem incorporadas ao treinamento da residência dos médicos de família ${ }^{94}$ : influência cultural nas convicções e nas escolhas sobre saúde; bases teóricas e filosóficas das abordagens em CAM; indicações e potenciais efeitos adversos de cada tratamento; evidências científicas de eficácia e custo-benefício de cada modalidade.

Em 1996, surgiu na Universidade do Arizona uma nova proposta para a educação médica intitulada "medicina integrativa", definida como uma "medicina que enfatiza a relação médico-paciente e integra o melhor da CAM com o melhor da medicina convencional", englobando aspectos humanísticos, preventivos e curativos dos diversos modelos terapêuticos existentes. A proposta da medicina integrativa é criar uma forma em que médicos convencionais e não convencionais possam trabalhar juntos, de forma confortável, em prol da melhora de seus pacientes.

$\mathrm{O}$ Program in Integrative Medicine (PIM) ${ }^{82}$ inclui dois anos de bolsas de estudo para quatro médicos residentes com média de seis anos de experiência clínica prévia. O primeiro ano do programa é dividido em três módulos didáticos: "fundamentos filosóficos", "práticas de estilo de vida" (promoção e prevenção à saúde) e "diversos sistemas terapêuticos" (fitoterapia, medicina manual, medicina chinesa, homeopatia, medicina energética e alopatia). No segundo ano, os residentes passam por quatro módulos de aprimoramento, compostos por "integração clínica" (aplicação do conhecimento teórico na prática clínica), "reflexão e desenvolvimento pessoal", "educação em pesquisa" e "liderança". Os residentes devem selecionar uma terapêutica não convencional para treinamento adicional no segundo ano, sendo estimulados a experimentar em si próprios as modalidades que recomendam a seus pacientes. Na adaptação da medicina integrativa ao currículo da residência médica convencional, cada residente dedica 8-10 horas semanais ao estudo de CAM, contabilizando mil horas de instrução no programa de dois anos.

Na Bruce Rappaport Faculty of Medicine (Haifa, Israel $)^{95}$ foi instituído um curso eletivo de introdução à CAM, dirigido a residentes e especialistas do Departamento de Medicina de Família, onde são ensinadas fitoterapia, medicina tradicional chinesa, homeopatia e medicina nutricional, em quatro módulos com 16 sessões (quatro sessões/módulo/terapêutica). Esta iniciativa induziu uma mudança positiva na atitude e no interesse dos médicos em relação à CAM baseada em evidências, fazendo com que passassem a recomendá-la a seus pacientes, empregando-a também em si próprios e seus familiares.

Analisando as diversas iniciativas desenvolvidas na área nas últimas décadas, autores discutem o que deve ser ensinado em um curso de CAM e como ministrá-lo, buscando traçar diretrizes para que os programas sejam aprimorados e atinjam seus objetivos ${ }^{96-101}$.

No Brasil, os programas para residência médica em homeopatia e acupuntura foram aprovados pela Comissão Nacional de Residência Médica (CNRM) em 2002 (Resolução CFM nº 1634/2002), com acesso direto e dois anos de duração (R1 e R2). Enquanto a residência em homeopatia é oferecida apenas no Hospital Universitário Gaffrée e Guinle (Universidade Federal do Estado do Rio de Janeiro, UNIRIO) ${ }^{102}$, a acupuntura é oferecida, atualmente, em nove instituições ${ }^{103}$ : Faculdade de Medicina da Universidade de São Paulo, Faculdade de Medicina de São José do Rio Preto, Hospital das Clínicas da Universidade Federal de Pernambuco, Hospital de Base do Distrito Federal, Hospital do Servidor Público Estadual Francisco Morato Oliveira (SP), Hospital Regional Homero de Miranda Gomes São José (SC), Hospital Universitário Prof. Polidoro Ernani de São Thiago (UFSC), Universidade Federal de São Paulo e Universidade Federal da Bahia.

De maneira geral, o ensino médico da homeopatia e da acupuntura no Brasil ocorre há décadas sob a forma de cursos de especialização ou pós-graduação lato sensu, ministrados pelas entidades formadoras ${ }^{104,105}$ e com carga horária em torno de 1.200 horas-aula, capacitando os médicos a prestarem as provas de títulos de especialista junto ao convênio das entidades de classe com o CFM e a Associação Médica Brasileira (AMB) ${ }^{106}$.

\section{DISCUSSÃO}

Terapêuticas não convencionais como a homeopatia e a acupuntura têm se apresentado como uma opção de tratamento para as mais diversas enfermidades humanas, sendo procuradas, nos últimos anos, por um contigente crescente da população. Embora apresentem aplicação clínica ampla e secular (milenar, no caso da acupuntura), sejam reconhecidas como especialidades médicas, estejam disponibilizadas em alguns serviços públicos de saúde(SUS), tenham suas consultas e procedimentos reembolsados pelas empresas de seguro médico e apresentem pressupostos científicos fundamentados no campo das pesquisas básicas e clínicas ${ }^{107-110}$, o desconhecimento de seus aspectos fundamentais pela classe médica resulta em preconceitos e críticas infundadas, perpetuadas pela ausência do ensino na grade curricular das faculdades de medicina.

No intuito de superar estes entraves, a homeopatia e a acupuntura deveriam estar inseridas no currículo de todas as faculdades de medicina brasileiras, na forma de disciplinas obrigatórias e eletivas. Considerando a complexidade e a diversidade dessas práticas, a carga 
horária para uma disciplina teórica deveria corresponder a um mínimo de 30 horas-aula ( 2 créditos), trazendo aos estudantes conhecimento suficiente para aconselharem seus futuros pacientes no uso dessas terapêuticas. Essas mesmas disciplinas também poderiam ser disponibilizadas nos programas de pós-graduação e residência médica. Ambulatórios e ligas acadêmicas deveriam ser oferecidos em conjunto com as disciplinas teóricas, para permitir uma vivência clínica e terapêutica aos estudantes.

Ampliando a oferta para a pós-graduação lato sensu e a residência médica específica nestas modalidades, segundo cargas horárias e programas específicos, as evidências científicas que respaldem estes modelos terapêuticos deveriam estar presentes em todas as iniciativas, por traduzirem para a linguagem acadêmica pressupostos e concepções distintos dos usualmente ensinados, facilitando a compreensão dos aprendizes.

No entanto, de forma análoga à necessidade de um número expressivo de médicos homeopatas e acupunturistas para atuarem nas redes públicas e privadas de assistência, suficiente para responder à demanda reprimida de pacientes que buscam estas terapêuticas, professores e pesquisadores habilitados nessas áreas também deveriam fazer parte do corpo docente das faculdades de medicina, para que propostas de ensino, pesquisa e assistência possam ser elaboradas e viabilizadas. A união destes profissionais em departamentos específicos permitiria a troca de experiências e a execução dessas iniciativas.

Exemplo desse tipo de organização ocorre na Escola de Medicina e Cirurgia da UNIRIO, instituição pioneira no ensino acadêmico da homeopatia, que possui um Departamento de Homeopatia e Terapêutica Complementar constituído por professores habilitados (médicos homeopatas) e contratados, encarregados pelo ambulatório didático e pelas disciplinas Matéria Médica Homeopática (obrigatória, 30 horas-aula, 2 créditos), Clínica Homeopática (optativa, 30 horas-aula, 2 créditos) e Terapêutica Homeopática (optativa, 30 horas-aula, 2 créditos) ${ }^{111,112}$. A partir de 2004, essa estrutura permitiu o oferecimento da residência médica em homeopatia.

Pelo fato de a acupuntura e a homeopatia serem utilizadas de forma adjuvante no tratamento de uma infinidade de enfermidades modernas, causa perplexidade a ausência destas especialidades na composição das equipes médicas nos diversos setores da saúde. Praticamente, todos os ambulatórios e enfermarias deveriam contar com a aplicação destas alternativas terapêuticas, minimizando o sofrimento dos pacientes, melhorando a resolutividade das doenças e diminuindo os custos e os efeitos colaterais dos tratamentos convencionais. Infelizmente, esses aspectos não são valorizados por coordenadores de cursos médicos e gestores de políticas em saúde.

Respostas a esse 'paradoxo' foram sugeridas em análise dos fatores facilitadores e dificultadores para a implantação de políticas públicas em homeopatia, realizada junto a gestores dos municípios de São Paulo ${ }^{113}$. Como aspectos 'facilitadores', foram citados: presença de médicos homeopatas na rede, demanda de usuários interessados pela homeopatia, disposição e aceitação do gestor à homeopatia e suas propostas, baixo custo dos medicamentos homeopáticos e existência de serviços de referência capazes de acolher a terapêutica homeopática. Dentre os 'dificultadores', teríamos: contratação de médicos homeopatas (principal dificuldade), influência de instância superior contrária à homeopatia, prioridade para as necessidades básicas da saúde municipal antes de instituir novos projetos, aquisição e fornecimento dos medicamentos, tempo utilizado nas consultas homeopáticas e o desconhecimento da racionalidade médica homeopática.

Em 2006, o Ministério da Saúde criou a Política Nacional de Práticas Integrativas e Complementares (PNPIC, Portaria MS No 971/2006) ${ }^{114}$, garantindo acesso 'parcial' da população às terapêuticas não convencionais. Com a PNPIC, os pacientes têm acolhimento gratuito nas áreas de Medicina Tradicional Chinesa/Acupuntura, Homeopatia, Plantas Medicinais e Fitoterapia, disponibilizadas nas Unidades Básicas de Saúde (UBS) e nos Núcleos de Apoio à Saúde da Família (NASFs), além dos hospitais.

Com resultados tímidos perante o enorme interesse por estas práticas, os efeitos desta política ilustram a importância que deveria ser dada ao ensino médico destas abordagens complementares em saúde: em 2007, após a implantação da PNPIC, foram realizados 97.240 procedimentos de acupuntura e, no ano seguinte, este número aumentou para 216.616 , com um crescimento de $122 \%$. No caso da homeopatia, em 2007, foram realizadas 312.533 consultas $^{115}$. Vale ressaltar que, nessa época, a homeopatia estava disponibilizada na rede pública em apenas $2 \%$ dos municípios brasileiros e em escassas iniciativas.

Traçando o panorama da educação médica em CAM, esperamos estimular a discussão sobre a importância das escolas médicas brasileiras em se adequarem às crescentes demandas nessa área, pois o ensino, a pesquisa e a assistência em CAM vêm ao encontro das necessidades da sociedade, que se interessa por novas formas de tratamento, mas não conhece os pressupostos científicos que respaldam a utilização correta e segura das mesmas.

\section{REFERÊNCIAS}

1. National Institute of Health. National Center for Complementary and Alternative Medicine (NCCAM).
Betseda, MD: NIH; 2013. Available from: http://nccam. nih.gov/. 
2. Scheffer M. Demografia médica no Brasil. v.2. Cenários e indicadores de distribuição. São Paulo: CREMESP/CFM; 2013. Disponível em: http://portal.cfm.org.br/images/stories/ pdf/demografiamedicanobrasil_vol2.pdf.

3. Teixeira MZ, Lin CA, Martins MA. O ensino de práticas não-convencionais em saúde nas faculdades de medicina: panorama mundial e perspectivas brasileiras. Rev Bras Educ Med. 2004;28(1):51-60. Disponível em: http://dx.doi. org/10.1590/S0100-55022007000100003

4. Teixeira MZ, Lin CA, Martins MA. Homeopathy and acupuncture teaching at Faculdade de Medicina da Universidade de São Paulo: the undergraduates' attitudes. Sao Paulo Med J. 2005;123(2):77-82. http://dx.doi. org/10.1590/S1516-31802005000200009.

5. Teixeira MZ. Homeopatia: desinformação e preconceito no ensino médico. Rev Bras Educ Med. 2007;31(1):15-20. http://dx.doi.org/10.1590/S0100-55022007000100003.

6. Teixeira MZ. Possíveis contribuições do modelo homeopático à humanização da formação médica. Rev Bras Educ Med. 2009;33(3):454-63. http://dx.doi.org/10.1590/S010055022009000300016 .

7. Amadera JE, Pai HJ, Hsing WT, Teixeira MZ, Martins MA, Lin CA. The teaching of acupuncture in the University of São Paulo School of Medicine, Brazil. Rev Assoc Med Bras. 2010;56(4):458-61. http://dx.doi.org/10.1590/S010442302010000400020 .

8. Eisenberg DM, Kessler RC, Foster C, Norlock FE, Calkins DR, Delbanco TL. Unconventional medicine in the United States. Prevalence, costs and patterns of use. N Eng J Med. 1993;328(4):246-52. http://dx.doi.org/10.1056/ NEJM199301283280406.

9. Eisenberg DM, Davis RB, Ettner SL, Appel S, Wilkey S, Van Rompay M, et al. Trends in alternative medicine use in the United States, 1990-1997: results of a follow-up national survey. JAMA. 1998;280(18):1569-75. http:// dx.doi.org/10.1001/jama.280.18.15.

10. Tindle HA, Davis RB, Phillips RS, Eisenberg DM. Trends in use of complementary and alternative medicine by US adults: 1997-2002. Altern Ther Health Med. 2005;11(1):42-9. Available from: http://goo.gl/TH5bzC

11. Fisher P, Ward A. Complementary medicine in Europe. BMJ. 1994;309(6947):107-11. http://dx.doi.org/10.1136/ bmj.309.6947.107.

12. Burg MA, Hatch RL, Neims AH. Lifetime use of alternative therapy: a study of Florida residents. South Med J. 1998;91(12):1126-31. Available from: http://web.a.ebscohost. com/ehost/pdfviewer/pdfviewer?sid=d9c22b62-708d-47b79f07-294fc48ce009\%40sessionmgr4003\&vid=2\&hid $=4$ 206

13. Barnes PM, Bloom B, Nahin RL. Complementary and alternative medicine use among adults and children: United States, 2007. Natl Health Stat Report. 2008;(12):1-23.

14. Furnham A, Forey J. The attitudes, behaviors, and beliefs of patients of conventional vs complementary (alternative) medicine. J Clin Psychol. 1994;50(3):458-69. Available from : http://web.b.ebscohost.com/ehost/pdfviewer/ pdfviewer?sid=cddc8bf4-4840-4231-a364-8e3ec661b343 $\% 40$ sessionmgr $110 \&$ vid $=2 \&$ hid $=114$

15. Veeramah EK, Holmes S. Complementary therapy: complement or threat to modern medicine? J R Soc Health. 2000;120(1):42-6. http://dx.doi. org 10.1177/146642400012000117.

16. Astin JA. Why patients use alternative medicine: results of a national survey. JAMA. 1998;279(19):1548-53. http:// dx.doi.org10.1001/jama.279.19.1548.

17. Mendicelli VLSL. Homeopatia: percepção e conduta de clientela de postos de saúde de São Paulo [tese]. São Paulo: Faculdade de Saúde Pública, Universidade de São Paulo; 1994.

18. Moreira GN. Homeopatia em Unidade Básica de Saúde (UBS): um espaço disponível [dissertação]. São Paulo: Faculdade de Saúde Pública, Universidade de São Paulo; 1999. Disponível em: http://www.bvshomeopatia.org.br/ texto/hounidadeBasicaSaudePossivel_GilMoreira.htm

19. Adler SR, Fosket JR. Disclosing complementary and alternative medicine use in the medical encounter. J Fam Pract. 1999;48(6):453-8.

20. Saxe GA, Madlensky L, Kealey S, Wu DP, Freeman KL, Pierce JP. Disclosure to physicians of CAM use by breast cancer patients: findings from the Women's Healthy Eating and Living Study. Integr Cancer Ther. 2008;7(3):122-9. http://dx.doi.org10.1177/1534735408323081.

21. Giveon SM, Liberman N, Klang S, Kahan E. A survey of primary care physicians' perceptions of their patients' use of complementary medicine. Complement Ther Med. 2003;11(4):254-60. http://dx.doi.org/10.1016/S09652299(03)00114-6

22. Silverstein DD, Spiegel AD. Are physicians aware of the risks of alternative medicine? J Community Health. 2001;26(3):159-74. Doi: 10.1023/A:1010303528081

23. Vora CK, Mansoor GA. Herbs and alternative therapies: relevance to hypertension and cardiovascular diseases. Curr Hypertens Rep. 2005;7(4):275-80. Doi: 10.1007/s11906005-0025-0

24. Dantas F. Desinformação e deformação no ensino médico: a homeopatia no contexto da farmacologia médica. Rev Bras Educ Med. 1985;9(1):25-9.

25. White AR, Mitchell A, Ernst E. Familiarization with complementary medicine: report of a new course for primary care physicians. J Altern Complement Med. 1996;2(2):30714. Available from: http://online.liebertpub.com/doi/ pdfplus/10.1089/acm.1996.2.307

26. Gianesella EMF. Homeopatia nas escolas médicas: ensino, assistência e pesquisa no Estado de São Paulo. [Dissertação]. São Paulo: Faculdade de Saúde Pública, Universidade de São Paulo; 1998.

27. Straus SE. Complementary and alternative medicine: challenges and opportunities for American medicine. 
Acad Med. 2000;75(6):572-3. http://dx.doi.org/10.1053/ sonc. 2002.50002

28. Konefal J. The challenge of educating physicians about complementary and alternative medicine. Acad Med. 2002;77(9):847-50. Available from: file://C:/Users/ DBD-FMUSP/Downloads/The_Challenge_of_Educating Physicians_about.2\%20(1).pdf

29. Murdoch-Eaton D, Crombie H. Complementary and alternative medicine in the undergraduate curriculum. Med Teach. 2002;24(1):100-2. http://dx.doi. org10.1080/00034980120103423.

30. Frenkel M, Frye A, Heliker D, Finkle T, Yzaguirre D, Bulik $\mathrm{R}$, et al. Lessons learned from complementary and integrative medicine curriculum change in a medical school. Med Educ. 2007;41(2):205-13. http://dx.doi.org/10.1111/j.13652929.2006.02654.x.

31. Rakel DP, Guerrera MP, Bayles BP, Desai GJ, Ferrara E. CAM education: promoting a salutogenic focus in health care. J Altern Complement Med. 2008;14(1):87-93. http:// dx.doi.org10.1089/acm.2007.0562.

32. Hui KK, Zylowska L, Hui EK, Yu JL, Li JJ. Introducing integrative East-West medicine to medical students and residents. J Altern Complement Med. 2002;8(4):507-15. http://dx.doi.org10.1089/107555302760253711.

33. Park CM. Diversity, the individual, and proof of efficacy: complementary and alternative medicine in medical education. Am J Public Health. 2002;92(10):1568-72. Available from: http://www.ncbi.nlm.nih.gov/pmc/articles/ PMC1447280/pdf/0921568.pdf.

34. Kligler B, Maizes V, Schachter S, Park CM, Gaudet T, Benn $\mathrm{R}$, et al. Education Working Group, Consortium of Academic Health Centers for Integrative Medicine. Core competencies in integrative medicine for medical school curricula: a proposal. Acad Med. 2004;79(6):521-31. Available from: http://bravewell.org/content/pdf/cachim.pdf

35. Cardini F, Weixin H. Moxibustion for correction of breech presentation: a randomized controlled trial. JAMA. 1998;280(18):1580-4. http://dx.doi.org10.1001/ jama.280.18.1580.

36. Coyle ME, Smith CA, Peat B. Cephalic version by moxibustion for breech presentation. Cochrane Database Syst Rev. 2012;5:CD003928. http://dx.doi.org10.1002/14651858. CD003928.pub3.

37. Harris WS, Gowda M, Kolb JW, Strychacz CP, Vacek $\mathrm{JL}$, Jones PG, et al. A randomized, controlled trial of the effects of remote, intercessory prayer on outcomes in patients admitted to the coronary care unit. Arch Intern Med. 1999;159(19):2273-8. http://dx.doi.org10.1001/ archinte.159.19.2273.

38. Coruh B, Ayele H, Pugh M, Mulligan T. Does religious activity improve health outcomes? A critical review of the recent literature. Explore (NY). 2005;1(3):186-91. http:// dx.doi.org10.1016/j.explore.2005.02.001.

39. Dubey NP. Integrated medicine - many approaches, one service. World Health Forum. 1997;18(1):56-8. Available from: https:/extranet.who.int/iris/restricted/ bitstream/10665/54648/1/WHF_1997_18\%281\%29 p5658.pdf

40. Hilsden RJ, Verhoef MJ, Rasmussen H, Porcino A, DeBruyn JC. Use of complementary and alternative medicine by patients with inflammatory bowel disease. Inflamm Bowel Dis. 2011;17(2):655-62. http://dx.doi.org10.1002/ ibd.21360.

41. Skovgaard L, Nicolajsen PH, Pedersen E, Kant M, Fredrikson S, Verhoef M, et al. Use of complementary and alternative medicine among people with multiple sclerosis in the Nordic Countries. Autoimmune Dis. 2012;2012:841085. http://dx.doi.org10.1177/1403494813481646.

42. Norred CL, Zamudio S, Palmer SK. Use of complementary and alternative medicines by surgical patientes. J Am Assoc Nurse Anesth. 2000;68(1):13-8. Available from: https://www.aanadc.com/newsandjournal/Documents/ useofcomplementary_0200_p13-18.pdf

43. White A, Hayhoe S, Hart A, Ernst E. Adverse eventes following acupuncture: prospective survey of 32000 consultations with doctors and physiotherapists. BMJ. 2001;323(7311):485-6. Disponível em: http://aim.bmj.com/ content/19/2/84.long.

44. Fugh-Berman A. Herb-drug interactions. Lancet. 1999;355(9198):134-8. http://dx.doi.org10.1016/S01406736(99)06457-0.

45. Linzer M, Konrad TR, Douglas J, McMurray JE, Pathman DE, Williams ES, et al. Management care, time pressure, and physician job satisfaction: results from the physician worklife study. J Gen Intern Med. 2000;15(7):441-50. http:// dx.doi.org 10.1046/j.1525-1497.2000.05239.x.

46. Haas JS, Cook EF, Puopolo AL, Burstin HR, Cleary PD, Brennan TA. Is the professional satisfaction of general internists associated with patient satisfaction? J Gen Med. 2000;15(2):122-3. http://dx.doi.org10.1046/j.15251497.2000.02219.x.

47. Ernst E, Resch KL, Whie A. Complementary medicine. What physicians think of it: a meta-analysis. Arch Intern Med. 1995;155(22):2405-8. http://dx.doi.org10.1001/ archinte.1995.00430220059006.

48. Pirotta MV, Cohen MM, Kotsirilos V, Farish SJ. Complementary therapies: have they become accepted in general practice? Med J Aust. 2000;172(3):105-9. Available from: http://goo.gl/7EK6IL

49. Corbin Winslow L, Shapiro H. Physicians want education about complementary and alternative medicine to enhance communication with their patients. Arch Intern Med. 2002;162(10):1176-81. http://dx.doi.org10.1001/ archinte.162.10.1.

50. Sikand A, Laken M. Pediatricians' experience with and attitudes toward complementary/alternative medicine. Arch Pediatr Adolesc Med. 1998;152(11): 059-64. http://dx.doi. org10.1001/archpedi.152.11.1059.

51. van Haselen RA, Reiber U, Nickel I, Jakob A, Fisher PA. 
Providing complementary and alternative medicine in primary care: the primary care workers' perspective. Complement Ther Med. 2004;12(1):6-16. http://dx.doi.org/10.1016/S09652299(03)00140-7.

52. Flannery MA, Love MM, Pearce KA, Luan JJ, Elder WG. Communication about complementary and alternative medicine: perspectives of primary care clinicians. Altern Ther Health Med. 2006;12(1):56-63. Available from: http://web.a.ebscohost.com/ehost/pdfviewer/ pdfviewer?sid=38fd6db2-b912-421c-a6cd-15ae9c094921\% 40sessionmgr4005\&vid $=2 \&$ hid $=4112$

53. Brown S. Use of complementary and alternative medicine by physicians in St. Petersburg, Russia. J Altern Complement Med. 2008;14(3):315-9. http://dx.doi.org10.1089/acm.2007.7126

54. Stange R, Amhof R, Moebus S. Complementary and alternative medicine: attitudes and patterns of use by German physicians in a national survey. Altern Complement Med. 2008;14(10):1255-61. http://dx.doi.org10.1089/ acm.2008.0306.

55. Bjerså K, Stener Victorin E, Fagevik Olsén M. Knowledge about complementary, alternative and integrative medicine (CAM) among registered health care providers in Swedish surgical care: a national survey among university hospitals. BMC Complement Altern Med. 2012;12:42. http://dx.doi. org10.1186/1472-6882-12-42.

56. Längler A, Boeker R, Kameda G, Seifert G, Edelhäuser F, Ostermann T. Attitudes and beliefs of paediatric oncologists regarding complementary and alternative therapies. Complement Ther Med. 2013;21(Suppl 1):S10-9. http:// dx.doi.org10.1016/j.ctim.2012.02.006.

57. Andritzky W. Medical students and alternative medicine - a survey. Gesundheitswesen. 1995;57(6):345-8.

58. Hopper I, Cohen M. Complementary therapies and the medical profession: a study of medical students' attitudes. Altern Ther Health Med. 1998;4(3):68-73. Available from: http://goo.gl/ta616K

59. Yeo AS, Yeo JC, Yeo C, Lee CH, Lim LF, Lee TL. Perceptions of complementary and alternative medicine amongst medical students in Singapore--a survey. Acupunct Med. 2005;23(1):19-26. Disponível em: http://aim.bmj.com/ content/23/1/19.long.

60. Chaterji R, Tractenberg RE, Amri H, Lumpkin M, Amorosi SB, Haramati A. A large-sample survey of first- and secondyear medical student attitudes toward complementary and alternative medicine in the curriculum and in practice. Altern Ther Health Med. 2007;13(1):30-5. Available from: http:// goo.gl/DLguVY

61. Greiner KA, Murray JL, Kallail KJ. Medical student interest in alternative medicine. J Altern Complement Med. 2000;6(3):231-4. doi:10.1089/acm.2000.6.231.

62. RosenbaumME, NislyNL, Ferguson KJ, KligmanEW.Academic physicians and complementary and alternative medicine: an institutional survey. Am J Med Qual. 2002;17(1):3-9. http:// dx.doi.org10.1177/106286060201700102.
63. Frenkel M, Frye A, Heliker D, Finkle T, Yzaguirre D, Bulik R, et al. Lessons learned from complementary and integrative medicine curriculum change in a medical school. Med Educ. 2007;41(2):205-13. http://dx.doi. $\operatorname{org} 10.1177 / 106286060201700102$.

64. Loh KP, Ghorab H, Clarke E, Conroy R, Barlow J. Medical students' knowledge, perceptions, and interest in complementary and alternative medicine. J Altern Complement Med. 2013;19(4):360-6. http://dx.doi. org10.1089/acm.2012.0014.

65. Kemper KJ, Vincent EC, Scardapane JN. Teaching an integrated approach to complementary, alternative, and mainstream therapies for children: a curriculum evaluation. J Altern Complement Med. 1999;5(3):261-8. doi:10.1089/ acm.1999.5.261.

66. Prajapati SH, Kahn RF, Stecker T, Pulley L. Curriculum planning: a needs assessment for complementary and alternative medicine education in residency. Fam Med. 2007;39(3):190-4. Disponível em: http://www.stfm.org/ fmhub/fm2007/March/Sarita190.pdf.

67. Cohen JJ. Reckoning with alternative medicine. Acad Med. 2000 75(6):571. http://goo.gl/MFrzxZ

68. Frenkel M, Arye EB. The growing need to teach about complementary and alternative medicine: questions and challenges. Acad Med. 2001;76(3):251-4.

69. British Medical Association. Complementary medicine: new approaches to good practice. Londres: BMA; 1993.

70. Morgan D, Glanville H, Mars S, Nathanson V. Education and training in complementary and alternative medicine: a postal survey of UK universites, medical schools and faculties of nurse education. Comp Ther Med. 1998;6(2):64-70. http:// dx.doi.org/10.1016/S0965-2299(98)80077-0.

71. Barberis L, de Toni E, Schiavone M, Zicca A, Ghio R. Unconventional medicine teaching at the Universities of the European Union. J Altern Complement Med. 2001;7(4):33743. http://dx.doi.org10.1089/10762800152709679.

72. Ethics manual: fourth edition. Ann Int Med. 1998;128(7):57694. http://dx.doi.org10.7326/0003-4819-128-7-19980401000012.

73. Bhattacharya B. MD programs in the United States with complementary and alternative medicine education opportunities: an ongoing listing. J Altern Complement Med. 2000;6(1):77-90. Disponível em: http://online.liebertpub.com/ doi/pdfplus/10.1089/acm.2000.6.77

74. Carlston M, Stuart MR, Jonas W. Alternative medicine instruction in medical schools and family practice residency programs. Fam Med. 1997;24:559-62.

75. Wetzel MS, Eisenberg DM, Kaptchuck TJ. Courses involving complementary and alternative medicine at U.S. medical schools. JAMA. 1998;280(9):784-7. http://dx.doi.org10.1001/ jama.280.9.784.

76. Ruedy J, Kaufman DM, MacLeod H. Alternative and complementary medicine in Canadian medical schools: a survey. Can Med Assoc J. 1999;160(6):816-7. Disponível em: 
http://www.cmaj.ca/content/160/6/816.reprint.

77. Tsuruoka K, Tsuruoka Y, Kajii E. Complementary medicine education in Japanese medical schools: a survey. Complement Ther Med. 2001;9(1):28-33. http://dx.doi.org/10.1054/ ctim.2000.0415.

78. Brokaw JJ, Tunnicliff G, Raess BU, Saxon DW. The teaching of complementary and alternative medicine in U.S. medical schools: a survey of course directors. Acad Med. 2002;77(9):876-81. Available from: http://goo.gl/JNXkWu

79. Melchart D, Linde K, Weidenhammer W, Worku F, Wagner $\mathrm{H}$. The integration of natural healing procedures into research and teaching at German universities. Altern Ther Health Med. 1995;1(1):30-3.

80. Chi C. Integrating traditional medicine into modern health care systems: examining the role of Chinese medicine in Taiwan. Soc Sci Med. 1994;39(3):307-21.

81. Kim JS, Kim DH, Lee WK, Suh JS, Song KE, Kang BJ, et al. Possibility in unification of oriental and western medicine education by combination of educational curricula. Uisahak. 1999;8(2):269-77. Available from: http://medhist.kams. or.kr/1999/269.pdf.

82. Maizes V, Schneider C, Bell I, Weil A. Integrative medical education: development and implementation of a comprehensive curriculum at the University of Arizona. Acad Med. 2002;77(9):851-60. Available from: http://goo. $\mathrm{gl} / \mathrm{mCPYhE}$

83. Laken MP, Cosovic S. Introducing alternative/complementary healing to allopathic medical students. J Altern Complement Med. 1995;1(1):93-8.

84. Hui KK, Zylowska L, Hui EK, Yu JL, Li JJ. Introducing integrative East-West medicine to medical students and residents.J Altern Complement Med. 2002;8(4):507-15. http:// dx.doi.org10.1089/107555302760253711.

85. Straus SE. Complementary and alternative medicine: challenges and opportunities for American medicine. Acad Med. 2000;75(6):572-3.

86. Pearson NJ, Chesney MA. The CAM Education Program of the National Center for Complementary and Alternative Medicine: an overview. Acad Med. 2007;82(10):921-6. Available from: http://goo.gl/0bLkWh

87. Lee MY, Benn R, Wimsatt L, Cornman J, Hedgecock J, Gerik $\mathrm{S}$, et al. Integrating complementary and alternative medicine instruction into health professions education: organizational and instructional strategies. Acad Med. 2007;82(10):939-45. DOI: 10.1097/ACM.0b013e318149ebf8

88. Verhoef MJ, Brundin-Mather R. A national approach to teaching complementary and alternative medicine in Canadian medical schools: The CAM in UME Project. Proc West Pharmacol Soc. 2007;50:168-73.

89. Consortium of Academic Health Centers for Integrative Medicine (CAHCIM), 2013. Disponível em: http://www. imconsortium.org/.

90. Kim do Y, Park WB, Kang HC, Kim MJ, Park KH, Min $\mathrm{BI}$, et al. Complementary and alternative medicine in the undergraduate medical curriculum: a survey of Korean medical schools. J Altern Complement Med. 2012;18(9):870-4. http:// dx.doi.org10.1089/acm.2011.0179.

91. Centro de Acupuntura do Instituto de Ortopedia (HCFMUSP), 2013. Disponível em: http://www.acupunturista.net/school/45/ iot-centro-de-acupuntura-do-instituto-de-ortopedia-etraumatologia-fmusp/

92. Disciplina Optativa Fundamentos da Homeopatia (FMUSP), 2013. Disponível em: http://www.fm.usp.br/homeopatia/.

93. Salles SAC. A presença da homeopatia nas faculdades de medicina brasileiras: resultados de uma investigação exploratória. Rev Bras Educ Med. 2008;32(3):283-90. http:// dx.doi.org/10.1590/S0100-55022008000300002.

94. Kliger B, Gordon A, Stuart M, Sierpina V. Suggested curriculum guidelines on complementary and alternative medicine: recomendations of the Society of Teachers of Family Medicine Group on Alternative Medicine. Fam Med. 2000;32(1):30-3.

95. Ben-Arye E, Frenkel M. Between metaphor and certainty: teaching an introductory course in complementary medicine. Harefuah. 2001;140(9):855-9,893.

96. Marcus DM. How should alternative medicine be taught to medical students and physicians? Acad Med. 2001;76(3):224-9.

97. Sampson W. The need for educational reform in teaching about alternative therapies. Acad Med. 2001;76(3):248-50.

98. Frenkel M, Ben Arye E. The growing need to teach about complementary and alternative medicine: questions and challenges. Acad Med. 2001;76(3):251-4. Available from: http://goo.gl/nMAQ95

99. Ben-Arye E, Frenkel M. An approach to teaching physicians about complementary medicine in the treatment of câncer. Integr Cancer Ther. 2004;3(3):208-13. http://dx.doi. org10.1177/1534735404267749.

100. Frenkel M, Ben-Arye E, Hermoni D. An approach to educating family practice residents and family physicians about complementary and alternative medicine. Complement Ther Med. 2004;12(2-3):118-25. http://dx.doi.org/10.1016/j. ctim.2004.07.044.

101. Jonas WB, Eisenberg D, Hufford D, Crawford C. The evolution of Complementary and Alternative Medicine (CAM) in the USA over the last 20 years. Forsch Komplementmed. 2013;20(1):65-72. http://dx.doi.org10.1159/000348284.

102. Associação Médica Homeopática Brasileira (AMHB). Residência médica, 2013. Disponível em: http://www.amhb. org.br/conteudo/148/168/residencia-medica.html.

103. Colégio Médico Brasileiro de Acupuntura (CMBA). Formação, Residência médica, 2013. Disponível em: http:// www.cmba.org.br/formacao/residencia-medica.html.

104. Associação Médica Homeopática Brasileira (AMHB). Escolas formadoras, 2013. Disponível em: http://www.amhb.org.br/ conteudo/125/142/escolas-formadoras.html.

105. Colégio Médico Brasileiro de Acupuntura (CMBA). 
Teixeira MZ, Lin CA. Educação médica em terapêuticas não convencionais.

Formação, Cursos reconhecidos, 2013. Disponível em: http:// www.cmba.org.br/cursos-reconhecidos.html.

106. Associação Médica Brasileira (AMB). Atualização, Títulos de especialista, 2013. Disponível em: http://www.amb.org. br/teste/servicos/titulos_de_especialista.html.

107. Teixeira MZ. Homeopatia: ciência, filosofia e arte de curar. Rev Med (São Paulo). 2006;85(2):30-43. Disponível em: http://revistas.usp.br/revistadc/article/view/59211.

108. Chin AL, Wu TH, Hong JP. Acupuntura: uma modalidade terapêutica validada no arsenal terapêutico do médico atual. Rev Med (São Paulo). 2006;85(3):110-3. Disponível em: http://revistas.usp.br/revistadc/article/view/59221.

109. Chin AL, Wu TH, Hong JP. Acupuntura: prática baseada em evidências. Rev Med (São Paulo). 2008;87(3):162-5. Disponível em: http://revistas.usp.br/revistadc/article/ view/59074.

110. Teixeira MZ. Evidências científicas da episteme homeopática. Rev Homeopatia (São Paulo). 2011;74(1-2):33-56. Disponível em: http://www.aph.org.br/revista/index.php/aph/article/ view/61/79.

111. Universidade Federal do Estado do Rio de Janeiro (UNIRIO). Escola de Medicina e Cirurgia. Departamentos. Departamento de Homeopatia e Terapêutica Complementar, 2013. Disponível em: http://escolademedicinaecirurgia.weebly. com/o-curso.html.

112. Universidade Federal do Estado do Rio de Janeiro (UNIRIO). Escola de Medicina e Cirurgia. Grade curricular do curso de medicina. Disponível em: http://www4. unirio.br/escolademedicina/documentos/GRADE\%20 CURRICULAR\%20A\%20PARTIR\%20DE\%202009-2.pdf.

113. Galhardi WMP, Barros NF, Leite-Mor ACMB. A homeopatia na rede pública do Estado de São Paulo: facilitadores e dificultadores. Rev Bras Med Fam Comunidade. 2012;7(22). Disponível em: http://www.rbmfc.org.br/rbmfc/article/ view/413/454

114. Ministério da Saúde. Secretaria de Atenção à Saúde. Departamento de Atenção Básica. Política Nacional de Práticas Integrativas e Complementares no SUS. Disponível em: http:/dtr2001.saude.gov.br/sas/PORTARIAS/Port2006/ GM/GM-971.htm.

115. Ministério da Saúde. Portal da Saúde. Notícias: Acesso à medicina não convencional cresce no SUS, 2013. Disponível em: http://portal.saude.gov.br/portal/aplicacoes/noticias/ default.cfm?pg $=$ dspDetalheNoticia\&id_area $=124 \& C O$ NOTICIA $=11001$. 